\title{
PReS-FINAL-2121: Results for 6 minute walk values in healthy German children show similar results as from Britain
}

\author{
| Foeldvari \\ From 20th Pediatric Rheumatology European Society (PReS) Congress \\ Ljubljana, Slovenia. 25-29 September 2013
}

\section{Introduction}

6 minute walk is a primary outcome measure in therapeutic studies for patients with pulmonary hypertension. Currently we have a two of sets of data[1,2] regarding test results in the 6 minute walk test (6MWT) in healthy children with a large span in the norm values in the different age groups.

\section{Objectives}

Aim of the study was to establish norm values for healthy German children for the 6 Minute Walk Test.

\section{Methods}

The team of an occupational therapist and a study nurse were visiting schools. Permission from the parents was give before the test. Always just probands from one class were invited to participate. The test were performed according the international guidelines[3]. The demographic data of the probands were collected and the parents filled out a short survey regarding the physical activity and the health condition. Children with chronic diseases, which decrease the stamina were excluded.

\section{Results}

Up till now 611probands participated from the age 5 ot 14 years. 343 of the 611 were female. The mean 6 minute walk continuously increased with age (Table 1.). It correlated in the age groups with the BMI.

\section{Conclusion}

Our results are in the range of the patients from the UK published by Lammers et al [1] and are in significantly
Table 1

\begin{tabular}{llllll}
\hline $6(n=21)$ & 491.6 & 479.6 & $6(n=18)$ & 472.2 & 481.1 \\
\hline $7(n=42)$ & 494.2 & 487.2 & $7(n=26)$ & 487.9 & 476.9 \\
\hline $8(n=43)$ & 485.0 & 494.8 & $8(n=33)$ & 488.2 & 493.8 \\
\hline $9(n=31)$ & 512,0 & 520.1 & $9(n=49)$ & 492.0 & 504.5 \\
\hline $10(n=48)$ & 516.8 & 519.7 & $10(n=58)$ & 526.3 & 521.3 \\
\hline
\end{tabular}

lower range than in the Chinese population collected data by $\mathrm{Li}$ et al.[2]. This reflects the importance of this study to gain norm values for our patient population.

\section{Disclosure of interest}

None declared.

Published: 5 December 2013

\section{References}

1. Li AM, et al: Standard reference for the six-minute-walk test in healthy children aged 7 to 16 years. Am J Respir Crit Care Med 2007, 176(2):174-80.

2. Lammers $A E$, et al: The 6-minute walk test: normal values for children of 4-11 years of age. Arch Dis Child 2008, 93(6):464-8.

3. Society AT: ATS statement: guidelines for the six-minute walk test. Am J Respir Crit Care Med 2002, 166(1):111-7.

doi:10.1186/1546-0096-11-S2-P133

Cite this article as: Foeldvari: PReS-FINAL-2121: Results for 6 minute walk values in healthy German children show similar results as from Britain. Pediatric Rheumatology 2013 11(Suppl 2):P133. 